\title{
Carrier transport characteristics of H-terminated diamond films prepared using molecular hydrogen and atomic hydrogen
}

\author{
Jin-long Liu ${ }^{1)}$, Liang-xian Chen ${ }^{1)}$, Yu-ting Zheng ${ }^{1)}$, Jing-jing Wang $^{2)}$, Zhi-hong Feng ${ }^{2)}$, and Cheng-ming Li ${ }^{1)}$ \\ 1) Institute for Advanced Materials and Technology, University of Science and Technology Beijing, Beijing 100083, China \\ 2) Science and Technology on ASIC Laboratory, Hebei Semiconductor Research Institute, Shijiazhuang 050051, China \\ (Received: 30 November 2016; revised: 15 March 2017; accepted: 20 March 2017)
}

\begin{abstract}
The H-terminated diamond films, which exhibit high surface conductivity, have been used in high-frequency and high-power electronic devices. In this paper, the surface conductive channel on specimens from the same diamond film was obtained by hydrogen plasma treatment and by heating under a hydrogen atmosphere, respectively, and the surface carrier transport characteristics of both samples were compared and evaluated. The results show that the carrier mobility and carrier density of the sample treated by hydrogen plasma are 15 $\mathrm{cm}^{2} \cdot \mathrm{V}^{-1} \cdot \mathrm{s}^{-1}$ and greater than $5 \times 10^{12} \mathrm{~cm}^{-2}$, respectively, and that the carrier mobilities measured at five different areas are similar. Compared to the hydrogen-plasma-treated specimen, the thermally hydrogenated specimen exhibits a lower surface conductivity, a carrier density one order of magnitude lower, and a carrier mobility that varies from 2 to $33 \mathrm{~cm}^{2} \cdot \mathrm{V}^{-1} \cdot \mathrm{s}^{-1}$. The activated hydrogen atoms restructure the diamond surface, remove the scratches, and passivate the surface states via the etching effect during the hydrogen plasma treatment process, which maintains a higher carrier density and a more stable carrier mobility.
\end{abstract}

Keywords: H-termination; diamond film; surface conductivity; carrier mobility; plasma treatment

\section{Introduction}

High-frequency and high-power devices based on diamond have attracted broad research attention because of their excellent electrical properties [1]. A p-type conductive layer has been widely observed to form on the surface of H-terminated diamond exposed to the atmosphere. It results from an adsorbate in the atmosphere forming an electrochemical system on the H-terminated diamond surface according to the surface transfer doping model [2-4]. Thus far, extensive work has been carried out to increase the surface conductivity using different adsorbates [5-8]. For example, the hole concentration on an H-terminated diamond surface was dramatically increased to $2.3 \times 10^{14} \mathrm{~cm}^{-2}$ after exposure to $\mathrm{NO}_{2}$ gas [5]. When fullerene and $\mathrm{C}_{60} \mathrm{~F}_{48}$ were transferred onto $\mathrm{H}$-terminated diamond surfaces, the electrical conductivity of p-type channels was increased to $10^{-5}$ and $10^{-4} \Omega^{-1}$, respectively [6]. High-dielectric-constant dielectric materials based on $\mathrm{Al}_{2} \mathrm{O}_{3}$ and $\mathrm{HfO}_{2}$ have also recently been used as surface adsorbates to fabricate metal-insulator-semiconductor field-effect transistors (MISFETs), and the fabricated devices exhibited more stable performances than devices without adsorbates [7-8].

In addition to being affected by atmospheric exposure, the surface conductivity of H-terminated diamond films is also affected by the treatment conditions, as also observed in the case of graphene [9]. Generally, $\mathrm{H}$ atoms produced by microwave (MW) plasma or by thermal dissociation resulting from hot filament (HF) have been used to hydrogenate diamond surfaces. On the one hand, energetic $\mathrm{H}$ atoms may damage the diamond surface by anisotropic etching and adversely affect the surface conductivity. On the other hand, when the diamond surface is treated by MW spherical plasma or a plasma column produced by $\mathrm{HF}$, it may exhibit non-uniformity over large regions because of the uneven distribution of the plasma density and the electric-field intensity, possibly resulting in unstable surface conductivity.

By contrast, H-termination of a diamond surface can be easily achieved through thermal hydrogenation using mole-

Corresponding author: Cheng-ming Li E-mail: chengmli@mater.ustb.edu.cn (C) The Author(s) 2017. This article is published with open access at link.springer.com 
cular hydrogen, and a surface with uniform conductivity is expected to be obtained. Using the diffuse-reflectance infrared Fourier transform (DRIFT) technique, we observed that stretching modes of $\mathrm{C}-\mathrm{H}$ vibrations appeared in the spectra of the diamond surface after it was exposed to a hydrogen atmosphere at high temperatures [10]. Other researchers compared the surface conductivity of high-pressure high-temperature (HPHT) IIa single-crystalline diamonds with (100), (111), and (110) orientations before and after hydrogenation using molecular hydrogen at different temperatures [11-12]. The sample with (110) orientation exhibited the lowest surface sheet resistivity of less than $50 \mathrm{k} \Omega$, whereas the (100) oriented sample was less sensitive to thermal hydrogenation. However, the effect of surface roughness on the carrier transport characteristics was ambiguous. In fact, the literature contains few reports on the surface conductivity of H-terminated diamond obtained by thermal hydrogenation.

In this paper, to elucidate the effect of treatment methods on the surface conductivity of H-terminated diamond, samples from the same diamond film were treated by microwave hydrogen plasma and by heating under a hydrogen atmosphere. The surface conductivity of both samples was compared. The results, in combination with the results of surface roughness measurements, were used to analyze the effects of treatment methods on the carrier transport behavior and the reaction between the diamond surface and molecular hydrogen/atomic hydrogen in detail.

\section{Methods}

High-quality diamond thin films were prepared by direct current (DC) arc jet chemical vapor deposition (CVD), as proposed previously [13]. After the thin films were ground using a polycrystalline diamond disk rotating at high speed (above $1000 \mathrm{r} / \mathrm{min}$ ) for a period of time, a growth surface of the as-deposited diamond films with a roughness $\left(R_{\mathrm{a}}\right)$ less than $5 \mathrm{~nm}$ was obtained. The polished diamond films were then cut into $15 \mathrm{~mm} \times 15 \mathrm{~mm}$ samples using a laser. Before hydrogenation, all the samples were boiled in a solution of $\mathrm{H}_{2} \mathrm{SO}_{4}: \mathrm{HNO}_{3}=5: 1$ (volume ratio) to remove the non-diamond carbon resulting from the laser cutting and to arrange the surface groups into oxygen termination. The samples were then ultrasonically cleaned using deionized water, acetone, and ethanol, sequentially. To avoid oxygen and other contaminants in air, the samples were stored in ethanol before being placed into the microwave chamber or the resistance furnace. The MW hydrogenation process has been reported elsewhere [14]. The treatment was conducted for $15 \mathrm{~min}$, and we observed that the surface roughness depended on the MW conditions.

Thermal hydrogenation experiments were conducted under hydrogen protective atmosphere in a resistance furnace. To distinguish the effects of atomic hydrogen and molecular hydrogen, the sample was positioned sufficiently far from the resistance wire so that only molecular hydrogen rather than atomic hydrogen could reach the diamond surface. After the pressure in the furnace reached $5 \mathrm{kPa}$, the samples were heated to $800^{\circ} \mathrm{C}$ under a flowing hydrogen atmosphere. The thermal hydrogenation time was $1 \mathrm{~h}$; the samples were then cooled to room temperature in the hydrogen atmosphere.

The surface morphology of the treated diamond surfaces was compared using atomic force microscopy (AFM) and a surface profilometer. Attenuated total reflection Fourier transform infrared spectroscopy (ATR-FT-IR) was used to determine the $\mathrm{C}-\mathrm{H}$ bonding modes on both diamond surfaces. A Ge crystal was used as the total reflection material. The angle of incidence was $45^{\circ}$, and a resolution of $4 \mathrm{~cm}^{-1}$ was chosen. The surface conductivity of the $\mathrm{H}$-terminated diamond films was characterized via van der Pauw-Hall tests using an Accent HL5500 Hall system. The van der Pauw-Hall pattern is described elsewhere [13]. Au ohmic contacts were evaluated by the transmission line method (TLM), which revealed a low specific contact resistance of less than $10^{-5} \Omega \cdot \mathrm{cm}^{2}$ in the absence of annealing. The area for testing surface conductivity in the van der Pauw-Hall pattern was limited to $100 \mu \mathrm{m} \times 100 \mu \mathrm{m}$. In order to evaluate the homogeneity of surface conductivity on both the diamond film prepared by hydrogen plasma treatment and that prepared by heating under a hydrogen atmosphere, five points on each sample were randomly chosen for conductivity tests.

\section{Results}

\subsection{Surface morphology and profiles of the diamond treated by molecular and atomic hydrogen}

The surface morphologies of diamond films treated by hydrogen plasma under a heated hydrogen atmosphere are shown in Fig. 1. The diamond film surface became rougher after the hydrogen plasma treatment compared to the surface of the film heated under the hydrogen atmosphere. Other authors have observed that the surface roughness of diamond films first decreases and then increases with increasing treatment time [15]. In our experiments, the lowest roughness of $0.6 \mathrm{~nm}$ was obtained after hydrogen plasma treatment for $15 \mathrm{~min}$, as indicated by the scratches resulting 
from the polishing process disappearing after hydrogen plasma bombardment. For the sample treated under a hydrogen atmosphere, the surface was smooth; specifically, the

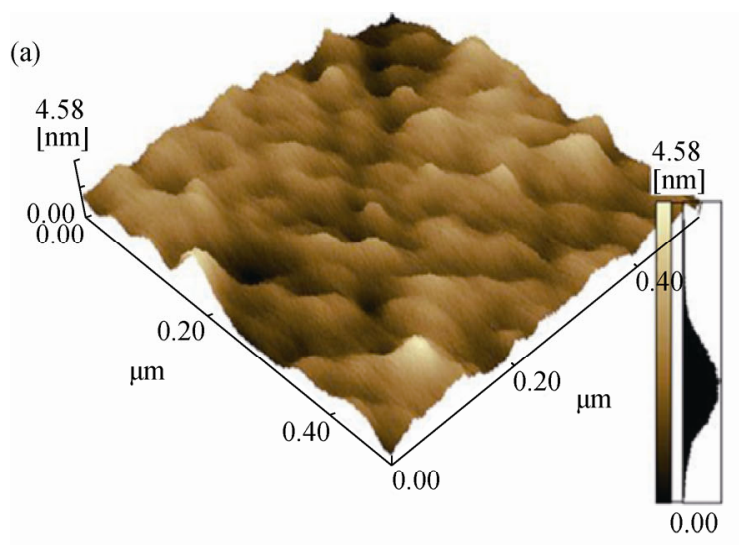

surface roughness was approximately $0.3 \mathrm{~nm}$ within a $1 \mu \mathrm{m} \times$ $1 \mu \mathrm{m}$ area, which is similar to the roughness of the as-polished surface.

(b)

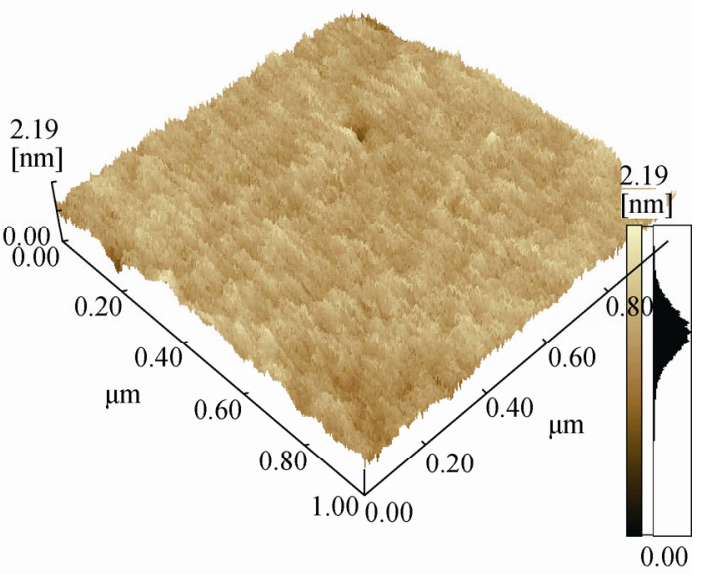

Fig. 1. Surface morphologies of diamond films treated by hydrogen plasma (a) and heated under a hydrogen atmosphere (b); the height information on the surface of both samples is shown in the color bars.

The 50- $\mu \mathrm{m}$ surface profiles of treated diamond films, as measured using a surface profilometer, are shown in Fig. 2. Many burrs in addition to some grooves are clearly observed on the film subjected to $1 \mathrm{~h}$ of thermal hydrogenation. These burrs and grooves result from variations in the size of the diamond abrasives used during the polishing process. At the earlier stage of polishing, diamond powders with a large particle size were used, which resulted in large scratches. As the particle size of the powder decreased, most of the diamond surface became smooth, although some large scratches remained. By contrast, in the profile of the diamond film surface subjected to the hydrogen plasma treatment for $15 \mathrm{~min}$, ups and downs but no burrs are observed, which is attributed to the hydrogen plasma etching effect.

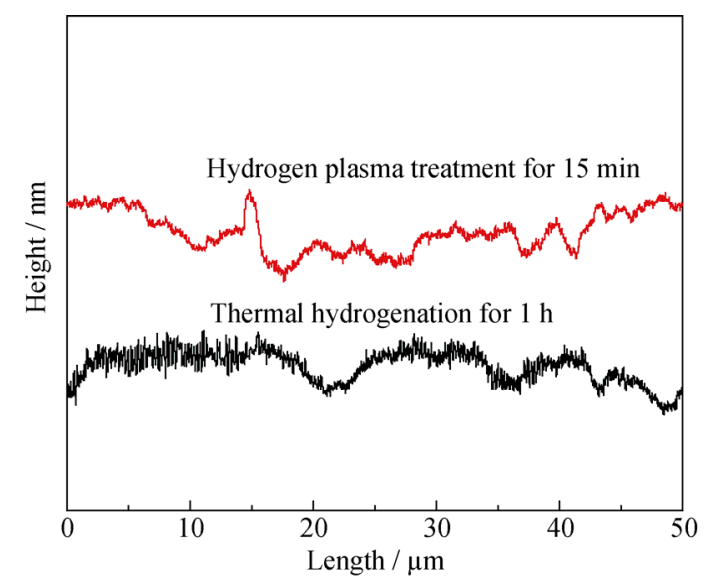

Fig. 2. Surface profiles of diamond films treated by hydrogen plasma and heated under a hydrogen atmosphere.

\subsection{Surface bonding of the diamond treated by molecu- lar and atomic hydrogen}

To compare the species on diamond surfaces treated by the two methods, we collected attenuated total reflection (ATR) spectra for both surfaces; the results are shown in Fig. 3. Before the hydrogenation treatment, the diamond surface consists of $\mathrm{C}=\mathrm{O}$ bonding corresponding to the bands near $1770 \mathrm{~cm}^{-1}$; this bonding results from oxidation due to the acid boiling step. After the hydrogenation treatment, the $\mathrm{C}=\mathrm{O}$ bonding almost disappears. Meanwhile, the $\mathrm{C}-\mathrm{H}$ stretching vibration bands corresponding to $\mathrm{CH}_{2}$ and $\mathrm{CH}_{3}$ groups are observed clearly in the spectra of the treated samples, which indicates the formation of H-termination on the diamond surface. The bands at 2850 and $2924 \mathrm{~cm}^{-1}$ are assigned to the symmetric stretching vibration of $\mathrm{CH}_{3}$ and to the asymmetric stretching vibration of $\mathrm{CH}_{2}$, respectively [16]. The $\mathrm{C}-\mathrm{H}$ stretching mode is related to the reconstruction of the diamond surface and varies with the diamond crystal orientation. In addition to these bands, the $\mathrm{OH}$ vibration bands at 1650 and $3000-3700 \mathrm{~cm}^{-1}$, which correspond to the stretching vibration and bending vibration of $\mathrm{H}_{2} \mathrm{O}$, respectively, are also observed; these bands are attributed to the ATR test being conducted in the atmosphere. The samples treated by hydrogen plasma and under hydrogen atmosphere show the similar stretching characteristics, which indicates that the samples exhibit similar orientations. By contrast, the intensity of the $\mathrm{C}-\mathrm{H}$ bonding band in the spectrum of the thermally hydrogenated diamond is stronger than that in the spectrum of the plasma hydrogenated sample. 


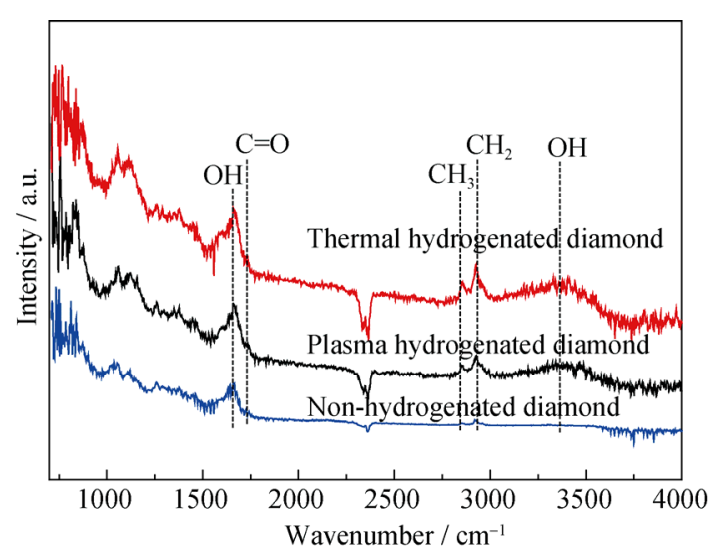

Fig. 3. ATR spectra of diamond films treated by hydrogen plasma and heated under a hydrogen atmosphere.

\subsection{Surface conductivity of the diamond treated by mo- lecular and atomic hydrogen}

After the hydrogenation treatments using hydrogen plasma and heating under a hydrogen atmosphere, we compared the surface conductivity of the diamond surfaces; the
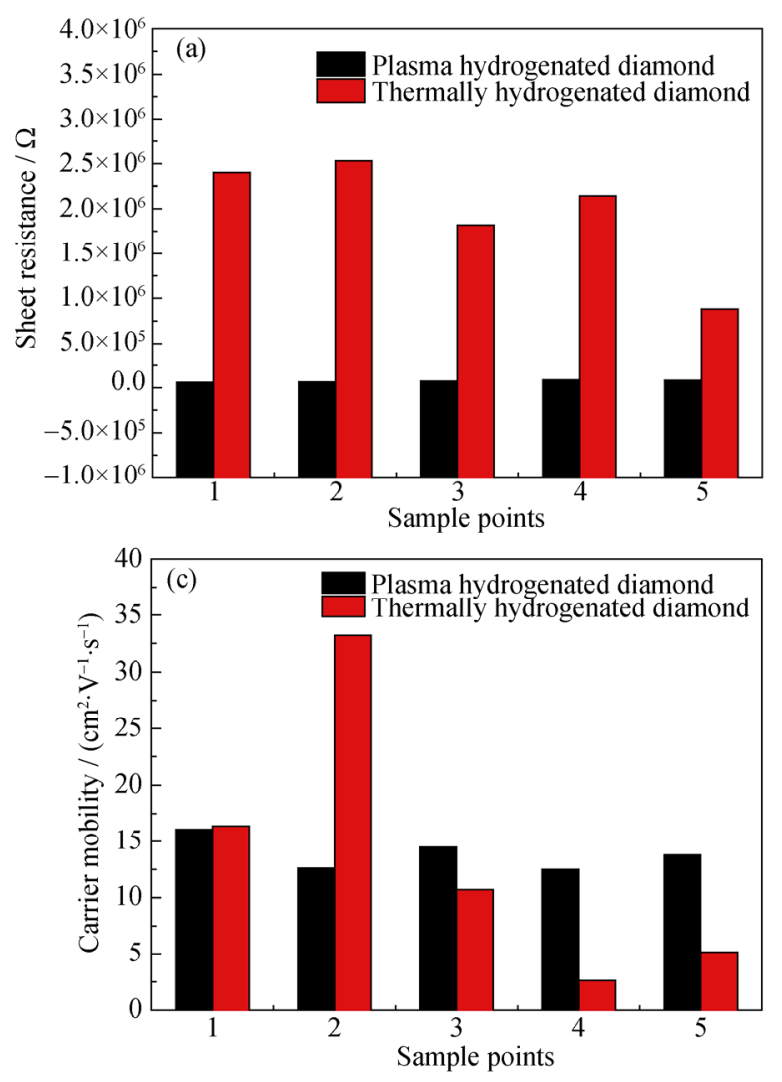

\section{Discussion}

\subsection{Carrier density difference analysis}

$\mathrm{H}$-termination on the diamond surface is necessary for p-type surface conductivity, which is necessary for diamond results are shown in Fig. 4. After hydrogenation treatments, both samples exhibit p-type conductivity. As shown in Fig. 4(a), after the hydrogen plasma treatment, the sheet resistance of the diamond surface is in the range from $6 \times$ $10^{4}$ to $8 \times 10^{4} \Omega$, which is common for diamond films prepared by DC arc jet CVD. In the case of the thermally hydrogenated samples, the sheet resistance is two orders of magnitude higher and reaches $1 \times 10^{6}$ to $2.5 \times 10^{6}$ $\Omega$. Meanwhile, the carrier density and carrier mobility in the conductive channel on both hydrogenated diamond surfaces are shown in Figs. 4(b) and 4(c). Compared with thermally hydrogenated diamond films, the plasma hydrogenated diamond films exhibit a carrier density greater than $5 \times 10^{12} \mathrm{~cm}^{-2}$, which is approximately one order of magnitude greater than that of the thermally hydrogenated films. With respect to the carrier mobility, the plasma hydrogenated films show similar values of approximately $15 \mathrm{~cm}^{2} \cdot \mathrm{V}^{-1} \cdot \mathrm{s}^{-1}$, whereas the values of the thermally hydrogenated films vary considerably from 2 to $33 \mathrm{~cm}^{2} \cdot \mathrm{V}^{-1} \cdot \mathrm{s}^{-1}$.

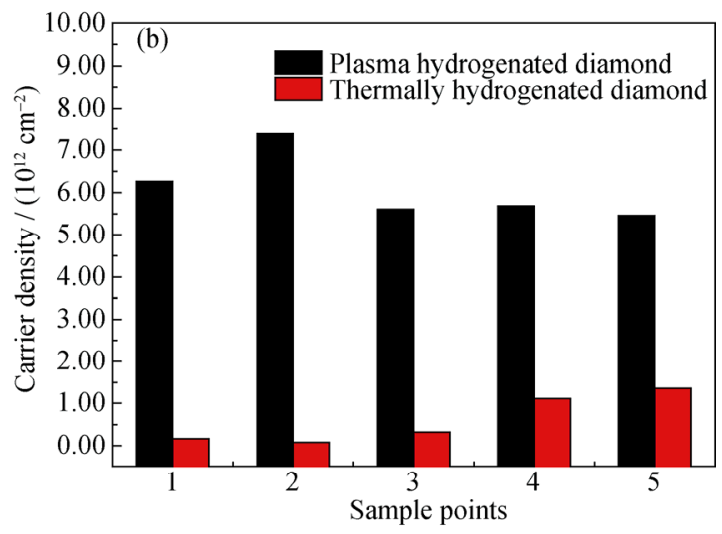

Fig. 4. Comparisons of the surface conductivity of plasma hydrogenated and thermally hydrogenated diamond films: (a) sheet resistance; (b) carrier density; (c) carrier mobility. films used in high-frequency and high-power electronic devices. We expected the thermally hydrogenated diamond films to exhibit more uniform surface conductivity and greater mobility compared to the plasma hydrogenated diamond films because the plasma caused less damage to the 
diamond surface. In our situation, H-termination on diamond surface was attained using either the hydrogen plasma treatment or the hydrogen atmosphere heat treatment, and we found that the plasma hydrogenated diamond exhibits substantially greater surface conductivity than the thermally hydrogenated diamond. Meanwhile, the carrier density of the plasma hydrogenated diamond surface is higher than that of thermally hydrogenated diamond, and the distribution of the carrier mobility is more uniform.

The carrier density in p-type conductive channel is known to be affected by the H-termination concentration on the diamond surface and to vary with the diamond orientation [17]. Because of the similar $\mathrm{C}-\mathrm{H}$ stretching vibration mode in the ATR spectra, we speculated that differences between diamond crystalline orientations of the hydrogen plasma treated and thermally treated samples are small because they are from the same diamond wafer. Meanwhile, we observed that the sample heated under a hydrogen atmosphere shows a higher $\mathrm{C}-\mathrm{H}$ band intensity but a lower carrier density compared to the plasma-treated sample. We confirmed that the formation of $\mathrm{H}$-termination on diamond surface resulted from the temperature controlled surface reaction between hydrogen atoms or molecules and carbon atoms [16]. Generally, the critical temperature is approximately $500^{\circ} \mathrm{C}$, at which the terminal $\mathrm{O}$ begins to desorb and leaves the carbon bond dangling. The samples were hydrogenated via plasma treatment for $15 \mathrm{~min}$ or via heat treatment for $1 \mathrm{~h}$ at $800^{\circ} \mathrm{C}$. Irrespective of the treatment method, the treatment time was sufficient in both cases, leading to a saturated carrier density [15]. In addition to the AFM and surface profile shown in Figs. 1 and 2, respectively, a schematic of both H-terminated diamond films prepared by the two methods is shown in Fig. 5. For the thermally hydrogenated sample, as shown in Fig. 5(a), the surface morphology with numerous burrs did not change after heat treatment under a hydrogen atmosphere; the $\mathrm{C}-\mathrm{O}$ bonding on the surface was simply converted into $\mathrm{C}-\mathrm{H}$ bonding. Although a higher $\mathrm{H}$-termination concentration was detected on the diamond surface because of the higher specific surface area, the holes in the channel generated on the basis of $\mathrm{C}-\mathrm{H}$ dipoles did not increase correspondingly.
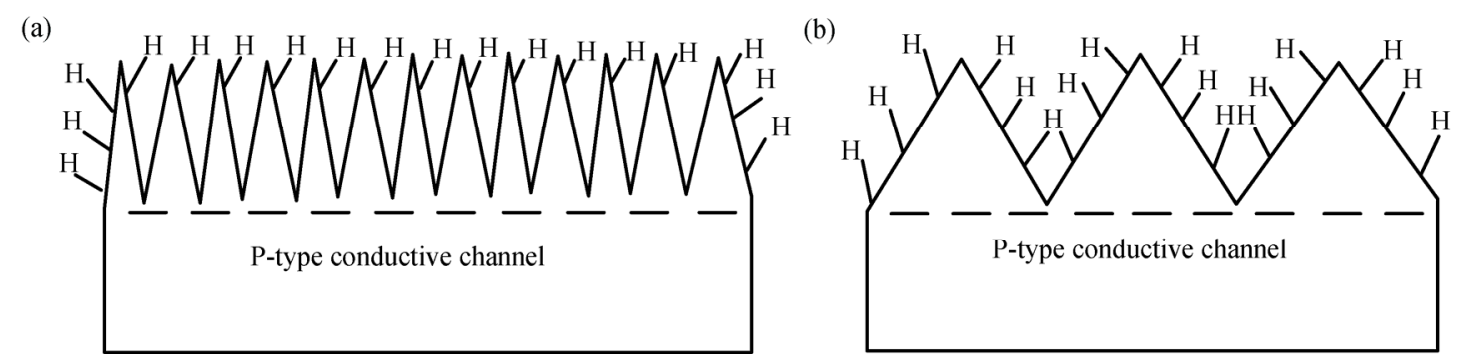

Fig. 5. Schematics of H-terminated diamond films prepared by heating under a hydrogen atmosphere (a) and hydrogen plasma treatment (b).

Excluding other factors such as impurities and crystalline orientation, the carrier density difference is attributed to electronic disorder present at the interface. On the basis of the simulation results, the carrier density in the conductive channel on a diamond surface has been reported to determine the depth of the wave function of holes into the adsorbate layer and the energy gap between the electrons in the adsorbate layer and holes in the channel [18]. In the case of a hole density of $5 \times 10^{12} \mathrm{~cm}^{-2}$, holes must overcome $6 \mathrm{meV}$; by contrast, in the case of a hole density one order of magnitude lower, the energy gap would be less than $1 \mathrm{meV}$, which indicates that the holes easily recombine with electrons in the thermally hydrogenated sample. That is, such a polished surface morphology with many burrs resulting from directed scratches is disadvantageous for improving the carrier density in the surface conductive channel. Furthermore, numerous electrically active surface states appear on the diamond surface after polishing; these states function as trapping centers, similar to vacancies or dangling bonds [19]. After the heat treatment under a hydrogen atmosphere, in addition to the conversion of $\mathrm{C}=\mathrm{O}$ bonding to $\mathrm{C}-\mathrm{H}$ bonding, some of the states on the diamond surface will be passivated, especially for the dangling bonds. However, because of the low activity of hydrogen molecules compared to hydrogen atoms, numerous surface states, including many vacancies, will remain unpassivated and will consume some of the holes generated in the conductive channel. By contrast, the diamond surface is etched off by activated hydrogen atoms during the hydrogen plasma treatment process. Not only does a chemical reaction occur between hydrogen atoms and diamond, but the distribution of states changes and most of the surface states are passivated, which stabilizes the carrier density. This stabilization is why a saturated carrier density is obtained by hydrogen plasma treatment for $5 \mathrm{~min}$ but not by heat treatment under hydrogen atmosphere for $1 \mathrm{~h}$. 


\subsection{Carrier mobility difference analysis}

We observed that the diamond surface obtained after hydrogen plasma treatment exhibits a stable carrier mobility of $15 \mathrm{~cm}^{2} \cdot \mathrm{V}^{-1} \cdot \mathrm{s}^{-1}$, whereas the carrier mobility of the thermally hydrogenated diamond varies in the range from 2 to $33 \mathrm{~cm}^{2} \cdot \mathrm{V}^{-1} \cdot \mathrm{s}^{-1}$ at different measurement points. Generally, the carrier mobility is affected by scattering centers in diamond films such as impurities and defects. In the case of the conductive channel on a diamond surface, the surface roughness scattering is one of the most important factors limiting the carrier mobility because of the two-dimensional hole gas characteristic. We speculated that a surface with lower roughness will exhibit greater carrier mobility. However, our results do not show such a tendency, possibly because of the surface profile randomness of polishing and the hydrogenation process. After polishing, the diamond surface shows random scratches and burrs at the microscale, and the subsequent thermal hydrogenation process weakly affects the surface morphology, which may increase the possibility of carrier scattering, especially at the sites of large scratches. This effect is why the surface mobility differs greatly among different areas. In the case of the plasma-treated surface, activated atoms restructure the surface carbon atoms and form the ordered surface via the etching effect. In particular, the disappearance of scratches was clearly observed after hydrogen plasma treatment for $15 \mathrm{~min}$, which may be the main reason for the nearly invariable carrier mobility.

\section{Conclusions}

In this paper, the surface conductive channel on samples from the same diamond film was obtained by hydrogen plasma treatment and by heating under a hydrogen atmosphere, respectively. The carrier transport characteristics of both samples were subsequently characterized and compared using van der Pauw-Hall tests. The conclusions are summarized as follows:

(1) The sample treated by hydrogen plasma exhibits a stable surface conductivity with a carrier mobility of $15 \mathrm{~cm}^{2} \cdot \mathrm{V}^{-1} \cdot \mathrm{s}^{-1}$ and a carrier density greater than $5 \times 10^{12}$ $\mathrm{cm}^{-2}$. By contrast, in the case of the thermally hydrogenated sample, the surface conductivity is lower; it exhibits an approximately one order of magnitude lower carrier density, and carrier mobility values that vary from 2 to $33 \mathrm{~cm}^{2} \cdot \mathrm{V}^{-1} \cdot \mathrm{s}^{-1}$.

(2) The conductivity differences between the two samples are attributed to the polished surface with random oriented scratches and burrs having more surface states and scattering centers; this surface is not improved by further thermal hydrogenation using hydrogen molecules.
(3) The activated hydrogen atoms restructure the diamond surface, removing the scratches and passivating the surface states via an etching effect during the hydrogen plasma treatment process, which may improve the carrier density and stabilize the carrier mobility.

\section{Acknowledgements}

This work was financially supported by the National Natural Science Foundation of China (No. 51402013), the China Postdoctoral Science Foundation (No. 2015T80037), and the Fundamental Research Funds for Central Universities (No. FRF-TP-15-052A2).

Open Access This article is distributed under the terms of the Creative Commons Attribution 4.0 International License (http://creativecommons.org/licenses/by/4.0/), which permits unrestricted use, distribution, and reproduction in any medium, provided you give appropriate credit to the original author(s) and the source, provide a link to the Creative Commons license, and indicate if changes were made.

\section{References}

[1] L.X. Chen, S. Liu, C.M. Li, Y.C. Wang, J.L. Liu, and J.J. Wei, Enhanced deposition of $\mathrm{ZnO}$ films by Li doping using radio frequency reactive magnetron sputtering, Int. J. Miner. Metall. Mater., 22(2015), No. 10, p. 1108.

[2] F. Maier, M. Riedel, B. Mantel, J. Ristein, and L. Ley, Origin of surface conductivity in diamond, Phys. Rev. Lett., 85(2000), No. 16, p. 3472.

[3] C. Verona, W. Ciccognani, S. Colangeli, F.D. Pietrantonio, E. Giovine, E. Limiti, M. Marinelli, and G. Verona-Rinati, Gate-source distance scaling effects in H-terminated diamond MESFETs, IEEE Trans. Electron Devices, 62(2015), No. 4, p. 1150.

[4] T. Yoshiteru, K. Shiraishi, M. Kasu, and H. Sato, Mechanism of hole doping into hydrogen terminated diamond by the adsorption of inorganic molecule, Surf. Sci., 609(2013), p. 203.

[5] H. Sato and M. Kasu, Maximum hole concentration for hydrogen-terminated diamond surfaces with various surface orientations obtained by exposure to highly concentrated $\mathrm{NO}_{2}$, Diamond Relat. Mater., 31(2013), p. 47.

[6] L. Ley, J. Ristein, F. Meier, and P. Strobel, Surface conductivity of the diamond: a novel transfer doping mechanism, Physica B, 376-377(2006), p. 262.

[7] J.W. Liu, M.Y. Liao, M. Imura, H. Osato, E. Watanabe, and Y. Koide, Electrical characteristics of hydrogen-terminated diamond metal-oxide-semiconductor with atomic layer deposited $\mathrm{HfO}_{2}$ as gate dielectric, Appl. Phys. Lett., 102(2013), art. No. 112910.

[8] M. Kasu, H. Sato, and K. Hirama, Thermal stabilization of 
hole channel on $\mathrm{H}$-terminated diamond surface by using atomic layer-deposited $\mathrm{Al}_{2} \mathrm{O}_{3}$ overlayer and its electric properties, Appl. Phys. Express, 5(2012), No. 2, art. No. 025701.

[9] Y.G. Shi, Y. Hao, D. Wang, J.C. Zhang, P. Zhang, X.F. Shi, D. Han, Z. Chai, and J.D. Yan, Effects of the flow rate of hydrogen on the growth of graphene, Int. J. Miner. Metall. Mater., 22(2015), No. 1, p. 102.

[10] T. Ando, M. Ishii, M. Kamo, and Y. Sato, Diffuse reflectance infrared Fourier-transform study of the plasma hydrogenation of diamond surfaces, J. Chem. Soc. Faraday Trans., 89(1993), No. 3, p. 1383.

[11] F. Fizzotti, A. Lo Giudice, C. Manfredotti, C. Manfredotti, M. Castellino, and E. Vittone, Diamond surface conductivity after exposure to molecular hydrogen, Diamond Relat. Mater., 16(2007), No. 4-7, p. 836.

[12] C. Manfredotti, F. Fizzotti, A. Lo Giudice, C. Manfredotti, M. Castellino, P. Bonino, and E. Vittone, A comprehensive study on hydrogenated diamond surfaces as obtained by using molecular hydrogen, Diamond Relat. Mater., 17(2008), No. 7-10, p. 1154.

[13] C.M. Li, J.L. Liu, L.X. Chen, J.J. Wei, L.F. Hei, J.J. Wang, Z.H. Feng, and H. Guo, An amazing semiconductor choice for high-frequency FET: H-terminated polycrystalline diamond film prepared by DC arc jet CVD, Phys. Status Solidi C, 11(2014), No. 11-12, p. 1692.

[14] J.L. Liu, C.M. Li, L.X. Chen, J.J. Wei, L.F. Hei, J.J. Wang,
Z.H. Feng, H. Guo, and F.X. Lv, Nucleation and growth surface conductivity of $\mathrm{H}$-terminated diamond films prepared by DC arc jet CVD, Diamond Relat. Mater., 32(2013), p. 48.

[15] J.L. Liu, C.M. Li, J.C. Guo, R.H. Zhu, L.X. Chen, J.J. Wei, L.F. Hei, J.J. Wang, Z.H. Feng, H. Guo, and F.X. Lv, Effect of atomic hydrogen bombardment on the surface conductivity of polycrystalline diamond films, Appl. Surf. Sci., 287(2013), p. 304.

[16] T. Ando, M. Ishii, M. Kamo, and Y. Sato, Thermal hydrogenation of diamond surfaces studied by diffuse reflectance Fourier-transform infrared, temperature-programmed desorption and laser Raman spectroscopy, J. Chem. Soc. Faraday Trans., 89(1993), No. 11, p. 1783.

[17] K. Hirama, H. Takayanagi, S. Yamauchi, J.H. Yang, H. Kawarada, and H. Umezawa, Spontaneous polarization model for surface orientation dependence of diamond hole accumulation layer and its transistor performance, Appl. Phys. Lett., 92(2008), No. 11, art. No. 112107.

[18] C.E. Nebel, B. Rezek, and A. Zrenner, Electronic properties of the 2D-hole accumulation layer on hydrogen terminated diamond, Diamond Relat. Mater., 13(2004), No. 11-12, p. 2031.

[19] V.I. Polyakov, N.M. Rossukanyi, A.I. Rukovishnikov, S.M. Pimenov, A.V. Karabutov, and V.I. Konov, Effects of post-growth treatment and coating with ultrathin metal layers on the band bending and field electron emission of diamond films, J. Appl. Phys., 84(1998), No. 5, p. 2882. 